\title{
11
}

\section{Interoperability Support by Integration of EXPRESS Models*}

\author{
Gerhard Scholz and Wolfgang Wilkes \\ University of Hagen, Praktische Informatik I \\ Feithstr. 140, D-58084 Hagen \\ Germany \\ e-mail: \{gerhard.scholz | wolfgang.wilkes\}@fernuni-hagen.de
}

\section{Introduction}

Interoperability of various tools is a major goal of the development of frameworks and design environments. Tools are supposed to work cooperatively together, i.e. they have to work on common data and to exchange their results. Unfortunately, quite often different tools work on different internal models of the world, the framework's database may provide an additional model, and if more than one framework is involved, it is very likely that it does provide yet another model.

One attempt to solve this problem is the provision of standardized information models (usually formulated by means of EXPRESS [EXP92]) which are used as a basis for data transfer (e.g. STEP file format) or for database accesses (e.g. via SDAI). These efforts are very important, but it is doubtful that the ultimate description of a domain within one information model can be achieved. Different standards will remain existing in the future, and every CAD domain has to be open for new applications and new technologies which will require new data. Thus, we will have to deal with a variety of tool internal models, framework schemas and even standards. Supporting the interoperability of information models or integrating them sets an important basis for the interoperability or integration of the tools, frameworks, and standards themselves.

One fundamental prerequisite to support interoperability on the level of the information model is to clearly identify the commonalities and differences of the models under consideration. On the first glance this seems to be an easy task. But in reality, this requires a lot of effort since the models usually describe common concepts from different perspectives using different modelling styles. Furthermore, besides the difficulty to identify the common structures it is also necessary to describe these common structures in an unambiguous way. Considering these aspects, the idea was born to define a core model which exactly describes the commonalities of the underlying information models.

\footnotetext{
* This work was supported by the EC in the context of the ESPRIT project 8370 ESIP
} 
A core model can only be of practical use if its relationships to the original models are described in a formal way. Only then it is possible to provide a common view of the overlapping areas of the models. Additionally, this sets the basis for the derivation of transformation programs which allow to transform data from one representation via the core model into another one. A further possible application could be the provision of an integrated view of the information content of both original sources (e.g. the integrated handling of VHDL and EDIF data).

The goal of this paper is to provide a methodology which allows to describe the common structures of different models and the way how they can be mapped to each other. Because we felt that the creation of a core model (schema intersection) and the creation of an integrated model (schema union) are strongly related problems, we started with inspecting the work which has been done in the area of schema integration for heterogeneous database systems (overviews can be found e.g. in [BLN86], [ACM91], and [IEEE91]). Our investigations showed that the models for electronic CAD comprise specific problems. Some of these problems are caused by EXPRESS since EXPRESS is not defined in an orthogonal way, i.e. a real world concept can be modelled in various ways. On the other hand, the models we are dealing with are far more complex than those considered in the heterogeneous database world. In particular, the entity types are much more interrelated, and it is not sufficient to only deal with single entities but we need to compare complex structures of entities and their relationships as a whole.

Our particular method has its roots in the concepts described in [SST92]. These give us a framework for describing the relationships of the core model to the original models in a formal way. In this paper, we will describe an adaptation of that method which has been tailored to the specific needs which arise in the engineering domains, more specifically which we were faced with during the process of developing a "core model" for EDIF and CFI. Some simplified examples from this core model [SchW94b] will illustrate our concepts. Here, we use EDIF always in the meaning of EDIF version 300 [EDIF93]. Similarly, $C F I$ is used as an abbreviation for CFI DR version 1.0.0 [CFI92]. When refering to entities of the CFI model, we will omit the prefix "cfidr".

The rest of this paper is organized as follows: In chapter 2, the basic idea of the core model creation is described. In chapter 3, this approach is extended to the "7-schemas-model" which provides the description of mappings between the original representations via the core model. In chapter 4, the situation of just partial overlapping concepts is discussed. Chapter 5 deals with the consideration of constraints. Chapter 6 concludes the paper.

\section{Creation of a Core Model}

The basic idea of our method corresponds to the concept of views for multi-objectbases as described in [SST92]. There, a same function is used to describe that objects from different objectbases represent the same real world entity. To these objects - more correctly: to their classes - views are created to unify attributes and relationships and to solve semantic heterogeneity. Finally, a union view of these views will be created which covers the common concepts. The presentation in [SST92] uses COCOON as the underlying object-oriented data model, where view declarations can be directly integrated into the class lattice. 


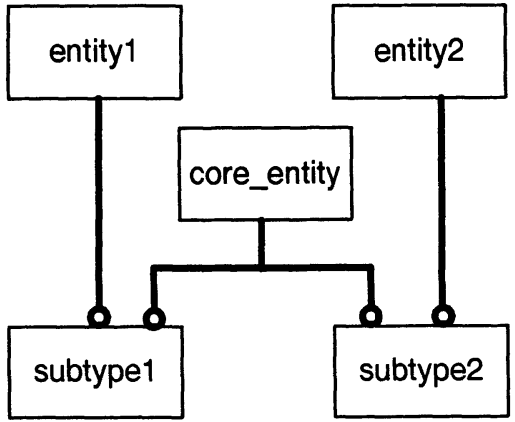

Fig. 1: Unification by a Core_Entity

Fig. 1 shows the modification of this concept for EXPRESS schemas. At first, a pair of entities - entity1 from schema A and entity 2 from schema B - will be identified which represent the same real world entity. By the definition of views, different representations of attributes and relationships will be adapted to each other. In EXPRESS this is done by means of derived attributes, which will be declared in subtypes. This step does not add new information, only already inherent information will become visible. Therefore these subtypes are semantically equivalent to their respective supertypes. Finally, the common attributes of these subtypes will be included in a common supertype core_entity. Thereby the core_entity unifies entityl and entity2.

Fig. 2 shows (simplified) excerpts from the information models of EDIF and CFI using the EXPRESS-G notation. In both models, a cell denotes an electronic chip, which can be described in different ways. A specific description is usually called view. In the following, we will consider just one view type, the netlist representation, which describes the electrical connections of the chips, called NetlistView in the CFI model and connectivity_view in the EDIF model. While CFI models this view by just one entity type, EDIF distinguishs between interface and implementation of the view. Furthermore, there exists the concept of a cluster, which gathers different views with the same interface.

EDIF:

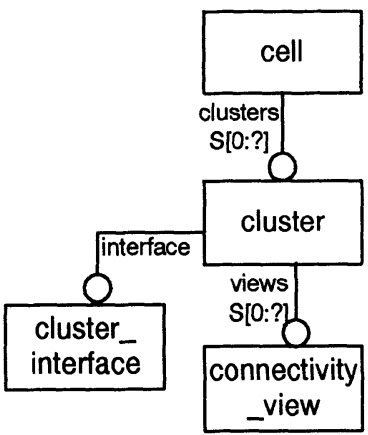

CFI:

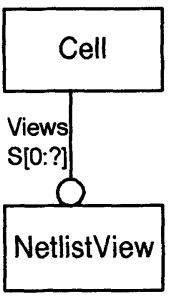

Fig. 2: Cell and View in EDIF und in CFI 
other direction. The question remains open how to represent the core model data within the original schemas. In example 1, for instance, there does not exist a unique straightforward solution for this. Cell and connectivity_view are not directly related in EDIF, but only indirectly via cluster, which does not exist in the core model. Thus, additional decisions have to be taken to map from the core model into an original schema.

The simpler example 2 demonstrates that this problem occurs, if the mapping from the original schema to the core model is not reversible. Wheras the PortInstScalar in the CFI model has one Props attribute, the same information in the EDIF entity type instance port is partitioned into two attributes new properties and overriding properties. The union of these set valued attributes leads from EDIF to the core model. To answer the question how CFI data can be represented in EDIF, also the direction from the core model back to EDIF needs to be defined. The additional specification of the mappings from the core model to the original schemas completes the 7-schemas-model.

\begin{tabular}{|c|c|}
\hline 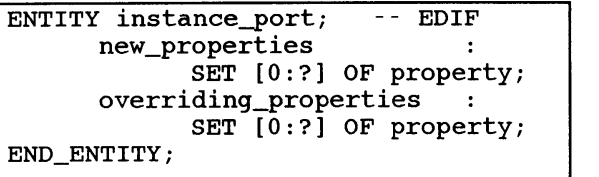 & $\begin{array}{l}\text { ENTITY PortInstScalar; } \\
\text { Props : SET [0:?] OF PFI } \\
\text { END_ENTITY; }\end{array}$ \\
\hline $\begin{array}{l}\text { ENTITY cm_instance_port; } \\
\text { properties : SET OF } \\
\text { END_ENTITY; }\end{array}$ & $\begin{array}{l}\text {-- Core_Model } \\
\text { m_property; }\end{array}$ \\
\hline $\begin{array}{l}\text { ENTITY ee_instance_port } \\
\text { SUBTYPE OF ( instance_port, } \\
\text { cm_instance_port); } \\
\text { DERIVE } \\
\text { SELF\Cm_instance_port.properties } \\
\text { : SET OF ee_property := } \\
\text { new_properties }+ \\
\text { overriding_properties; }\end{array}$ & $\begin{array}{l}\text { ENTITY ec_PortInstScalar } \\
\text { SUBTYPE OF ( PortInst, } \\
\text { cm_instance_port); } \\
\text { DERIVE } \\
\text { SELF\Cm_instance_port.properties } \\
\quad: \text { SET OF ec_Prop := Props; } \\
\text { END_ENTITY; }\end{array}$ \\
\hline
\end{tabular}

\section{Example 2}

In Fig. 3 the original schemas are named A and B and the core model is named C. The other schemas contain the subtypes which describe the respective transformation. The lines ending in a circle show, which schemas use information of other schemas. (EXPRESS offers interface specifications for this purpose.) The schemas A, B, and C can be used by themselves, whereas the transformation schemas, of course, need the information about source and target of the mapping. It should be observed, that at first with this approach the transformations to and from the core model can be defined independently of each other.

Example 2a complements example 2. For the mapping from the core model to EDIF, the properties of the core model will be attached to the attribute new properties. Of course, this is just one of several possibilities. But it is essential that the chosen solution is documented within a formal frame. 


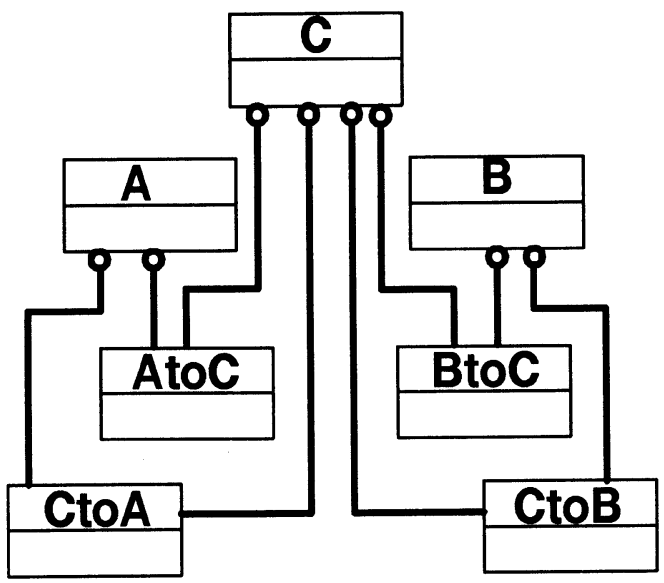

Fig. 3: The 7-schemas-model

(EXPRESS-G schema level diagram)

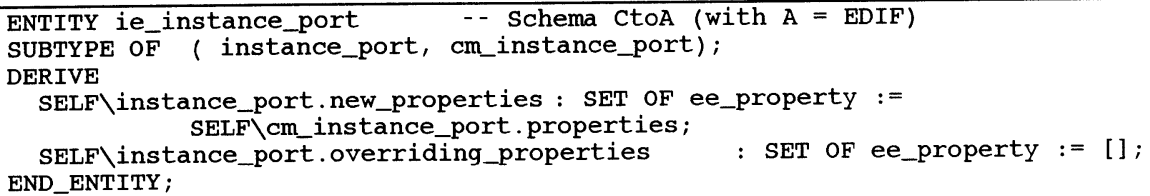

\section{Example 2a}

When data are transferred from an original schema to the core model and then back to the representation of the original schemas, they usually will not have their original shape. A transformation cycle via the core model changes overriding properties to new properties. That raises the question on the relationship between the mappings to and from the core model. Which information will be maintained, and which will be lost? The answer is obvious: the core model defines just the information which can be transferred without loss.

- Rule 1: A mapping from the core model into one of the original schema representations and back to the core model has always to be the identity function.

This restriction is not guaranteed by the 7 -schema-model itself. But it is a minimal requirement for the correctness of the mappings and the legitimate use of the term core model.

A further requirement can be explained using example 1: when mapping from the core model to EDIF, the core concept cm_connectivity_view will be mapped not only to the EDIF connectivity view, but some attributes will be mapped to cluster and to cluster_interface (fig. 2). Therefore schema CtoA (with $\mathrm{A}=\mathrm{EDIF}$ ) contains 3 subtypes of cm connectivity view, which describe the mapping of certain attributes to the corresponding EDIF concept. EXPRESS facilitates this way of description, because an object of a supertype may belong simultaneously to several subtypes. 
example 3 exactly this constraint is declared in the entity subtype ee_instance. In that case together with the cluster a unique view is specified, and the mapping to the core model remains deterministic.

\section{Consideration of Constraints}

The last section showed the need for constraint declarations in the subtypes describing the mappings. Beyond that, the core model entities themselves may need to be provided with constraints. But whereas an attribute will be included in the core model only, if it has a correspondence in both original schemas, a constraint needs to be defined if it occurs in just one original schema. This is a consequence of the fact that all core model data shall be representable in both original schemas. A core object which violates a constraint of an original schema cannot be transformed into this representation.

The 7-schemas-model does not provide a formal derivation of constraints for the core model. It has been defined to describe how objects in the representation of one schema are related and can be transformed into objects in the representation of another schema. But it does not define how the other schema (e.g. the core model) has been created. The definition of the core model, i.e. the definition of its entities, their attributes and relationships, and the definition of the constraints is a process which relies on domain knowledge and human decisions and cannot be fully automated. Support of this process by expert systems is feasible. But the development of such tools would be a research area in its own right and is not the topic of the current investigation.

In the present approach, the origin of the core model constraints is indicated by comments in the EXPRESS code. Example 4 shows an extended description of the core model entity cm cell of example 1 (also still simplified). An inverse attribute owner indicates that each cm_cell has to be used by exactly one library. This constraint has a correspondance in both original schemas, although it is expressed there in different ways. In EDIF, a domain rule is used to denote the cardinality constraint. Furthermore, a derived attribute (which relies on this domain rule) underlines the meaning of this direction of the relationship between cell and library. In CFI, an explicit attribute Owner is used. Two domain rules are needed to state that this attribute and the Cells attribute of the CFI Lib are inverse to each other. The unique rule of $\mathrm{cm}$ _cell requires that the cell name is unique within each library. The origin of this constraint is a domain rule in the CFI entity Lib.

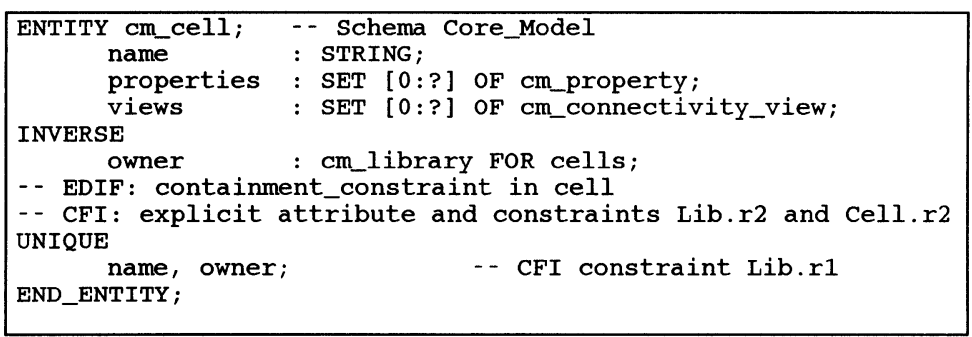

Example 4 
A detailed analysis of constraints in the information models of EDIF and CFI has been presented in [SchW94a]. A lot of these constraints can be replaced by specific structural EXPRESS mechanisms (INVERSE, UNIQUE, redeclaration). These structural equivalents are used for the core model as far as possible. For this purpose, the transformations described in [SchW94a] are applied in an informal way.

Although the derivation of the core model constraints is not visible in the 7-schemas-model, their existence is in no way arbitrary: when an object is transfered from one representation to another one, it has to obey the constraints of the target schema. Therefore when constraints are missing in the core model, there may exist objects whose transformation to an original schema representation would violate the constraints defined there. Such a counterexample would invalidate the given specification. On the other hand, when superfluous constraints are declared for the core model, the transformation capability is unnecessarily restricted which may impair an efficient use of the specification.

\section{Conclusion}

We have presented a formal frame, which allows to create a core model of given information models, which will describe the commonalities of the original models. In transformation schemas, subtypes will be defined which describe how to generate the core information from the original representations. Thereby a formal relationship is defined between the core model and the original schemas. A modification of the original schemas is not needed. If in addition the mappings from the core model to the original schemas will be defined, a transformation of data from one original representation into another one is possible via the core model. Thereby exactly those data can be mapped without loss, which are representable in the core model.

The concrete development of a core model for EDIF and CFI in [SchW94b] has proven that the approach is suitable for dealing with complex groups of entity types and their relationships. It allows to express even the case, that concepts of the original schemas only partially overlap. The inclusion of inheritance relationships (sub/supertypes) does not cause specific problems. This could not be shown here in detail. All constraints of the original models have to be included in the core model. Previous work [SchW94a] has given a classification of constraints and transformations of algorithmic constraints into a simpler structural form (UNIQUE and INVERSE clauses) for some frequently occuring constraint types.

A goal for our future research is the systematic analysis of the experiences of the concrete EDIF-CFI work to develop detailed rules for the unification work in the frame of the 7schemas-model. The translation of the transformation schemas into EXPRESS-M [EXPM94] representations as a first step to the implementation of translators is another open topic.

For the conceptual description, EXPRESS-M is not the first choice. The relationship between entities of the core and original models is less strong in EXPRESS-M, which would impair the readability of the description and would require the definition of additional rules to restrict arbitrary mappings. The aspect of the transformation describing subtype as a view of the original entity would get lost. It is not obvious whether the instance control of EXPRESS-M fulfills our needs when mapping relationships between entities. We simply refer to the subtype describing the transformation of the target entity or (when necessary in mappings from the core 
model) make use of the EXPRESS entity constructor. The dependency of the EXPRESS-M instance control on the order of the mapping statements indicates, that EXPRESS-M is more suited for an implementation description than for a conceptual description of relationships between schemas. A difficulty for a translation into EXPRESS-M may be that EXPRESS-M does not support the complex entity construction operator.

The basic idea of our methodology is derived from concepts for schema integration in the field of heterogeneous databases. The concepts presented above will surely play also an important role for the definition of an integrated view, which provides all information of the underlying schemas. Whereas the core model represents a generalization of the schemas $\mathrm{Ato} C$ and $\mathrm{BtoC}$ (fig. 3), the integrated view can be regarded as a common specialization of both AtoC and BtoC: the information derived for the core model creation as well as the original information will be passed on to the entities of the integrated view. It will be a topic of future research, whether the definition of the integrated view can be described by means of EXPRESS in a similar way (e.g. using multiple inheriting subtypes) as it is possible for the core model.

\section{References}

[ACM91] ACM SIGMOD Records, Special Issue on "Semantic Issues in Multidatabase Systems". Vol. 20, No. 4, December 1991.

[BLN86] C. Batini, M. Lenzerini, S.B. Navathe: A comparative analysis of methodologies for database schema integration. ACM Computing Surveys, Vol. 18., No. 4, December 1986.

[CFI92] CAD Framework Initiative, Inc.: Design Representation Programming Interface: Electrical Connectivity. Version 1.0.0, Austin, TX, November 1992.

[EDIF93] EDIF Electronic Design Interchange Format, Version 300 EIA-618. Electronic Industries Association, 2001 Pennsylvania Avenue NW, Washington D.C. 20006, USA, June 1993.

[EXP92] ISO DIS 10303-11. Product Data Representation and Exchange - Part 11: The EXPRESS Language Reference Manual. TC184/SC4 Document N 151. August 1992.

[EXPM94] Ian Bailey (ed.): EXPRESS-M Reference Manual. ISO TC184/SC4/WG5 Document N 51. May 1994.

[IEEE91] IEEE Computer, Special Issue on Heterogeneous Distributed Database Systems, December 1991.

[SchW94a] G. Scholz, W. Wilkes: Preliminary Report on Constraint Classification in Information Models. ESPRIT project 8370 ESIP, Document No. ESIP/FUHAGEN/001.1, April 1994.

[SchW94b] G. Scholz, W. Wilkes: Specification of a Core Model for Netlist. ESPRIT project 8370 ESIP, Document No. ESIP/FUHAGEN/004.1, October 1994.

[SST92] M. H. Scholl, H.-J. Schek, M. Tresch: Object Algebra and Views for MultiObjectbases. Proc. Int. Workshop on Distributed Object Management, Edmonton, Canada, August 1992. 